\title{
Assalto no local de trabalho, personalidade e coping: Uma revisão da literatura
}

\author{
Workplace robbery, personality and coping: A literature review
}

Andréia Mello de Almeida Schneider ${ }^{[a]}$, Blanca Susana Guevara Werlang ${ }^{[b]}$

\footnotetext{
[a] Psicóloga, mestre em Psicologia Clínica pela Pontifícia Universidade Católica do Rio Grande do Sul (PUCRS), Porto Alegre, RS - Brasil, e-mail: andreia.schneider@yahoo.com.br

${ }^{[b]}$ Psicóloga, doutora em Ciências Médicas e Saúde Mental pela Universidade Estadual de Campinas (Unicamp), professora titular da Faculdade de Psicologia da Pontifícia Universidade Católica do Rio Grande do Sul (PUCRS), Porto Alegre, RS Brasil, e-mail: bwerlang@pucrs.br
}

Recebido: 23/05/2011 Received: 05/23/2011

Aprovado: $14 / 09 / 2011$ Approved: 09/14/2011

\section{Resumo}

Trabalhadores de locais onde há grande circulação de pessoas e que lidam com grandes quantias de dinheiro são expostos a um contexto de potencial risco de assalto. Como sujeitos a violência interpessoal comunitária, tornam-se pessoas vulneráveis ao sofrimento psíquico advindo desse evento estressor. Diante disso, esta revisão de literatura visa a analisar aspectos da personalidade e as formas de enfrentamento do estresse por parte das vítimas. Trata-se de uma revisão sistemática sobre o tema assalto no local de trabalho e suas consequências psicológicas. 0 estudo foi baseado no levantamento da produção científica nas bases de dados PsycINFO, MEDLINE/PubMed e LILACS dos últimos quinze anos, utilizando diferentes combinações de unitermos. A maioria dos estudos identificados aponta a presença de sintomas psicopatológicos nas vítimas de assalto no local de trabalho. Além disso, quanto à personalidade, verificou-se, nesta revisão, estudos que assinalam a presença de evidências de baixa autoestima, dificuldades no relacionamento interpessoal e no afeto. Quanto à estratégia de enfrentamento, as pesquisas mostram indício de maior sofrimento nas vítimas de assalto que usavam estratégias diferentes de modo alternado. Nota-se a importância de se considerar a avaliação da personalidade após uma vivência de assalto no local de trabalho.

Palavras-chave: Personalidade. Coping. Assalto.

\section{Abstract}

Individuals who work in places with great movement of people and deal with large sums of money are exposed to an environment of potential risk of robbery. As they are subject to community interpersonal violence, they become vulnerable to the psychological suffering of this stressor. Hence, this systematic review aims to verify aspects of personality and ways of coping with stress by these victims. The study was based on the research of scientific production on the PsycINFO, MEDLINE/PubMed and LILACS databases over the last fifteen years, using different keywords combinations. Most of these studies indicate the presence of psychopathological symptoms in robbery victims in the workplace. Moreover, regarding personality, the presence of evidences of low self-esteem, difficulties in interpersonal relationships and affection was shown in this review. Regarding coping strategy, there was an indication of greater suffering on the victims who used 
different strategies in alternate ways. It seems clear that the importance of considering the assessment of personality after experiencing an assault in the workplace.

Keywords: Personality. Coping. Robbery.

\section{Introdução}

Notícias sobre a violência são diariamente divulgadas na mídia impressa e eletrônica. Isso evidencia que esse tipo de evento, mesmo sendo um fato antigo, continua presente em diversos setores da sociedade atual. No documento intitulado Relatório Mundial sobre a Violência e a Saúde (Krug, Dahlberg, Mercy, Zwi \& Lozano, 2002), a violência é apontada como um dos principais problemas de saúde pública no mundo e, para sua melhor compreensão, é subdividida em três grandes categorias: (1) autoinfligida; (2) interpessoal - comunitária ou familiar; e (3) coletiva - social, política ou econômica.

A primeira categoria é composta pelo "comportamento suicida" e "autoabuso" como, por exemplo, a automutilação. A segunda engloba a violência familiar, aquela praticada entre membros da mesma família, mas não necessariamente convivendo dentro da mesma casa, e a violência comunitária, que são atos aleatórios de violência, tais como os praticados nas ruas, escolas e locais de trabalho. Já a terceira categoria, a violência coletiva, pode ser de ordem: social, incluindo aí os crimes de ódio por grupos organizados como atos terroristas; política, a exemplo das guerras; ou ainda econômica, envolvendo ataques de grupos maiores motivados pelo ganho econômico.

Considerando, então, essa classificação, o evento de assalto no local de trabalho é compreendido como um ato de violência interpessoal comunitária. 0 Código Penal Brasileiro (Brasil, 2000), no qual a Polícia Civil baseia-se para aplicar as penalidades previstas em lei, não contempla a definição de "assalto", mas sim define "roubo" pelo artigo 157. Nele conceitualiza o termo como sendo o ato de "subtrair coisa móvel alheia, para si ou para outrem, mediante grave ameaça ou violência à pessoa" (p. 198). Essa definição se enquadra no que a sociedade em geral tende a chamar de assalto. No Manual Diagnóstico e Estatístico dos Transtornos Mentais - DSM-IV-TR (American Psychiatric Association, [APA], 2002). o assalto é definido como um evento traumático que envolve a experiência pessoal direta ou indireta (como testemunha ou por tomar conhecimento de ato violento vivenciado por amigo ou parente próximo) por parte do sujeito.

Alguns autores (Breslau, Chilcoat, Kessler \& Davis, 1999; Kaser-Boyd \& Evans, 2008) apontam que estudos de prevalência desenvolvidos por pesquisadores norte-americanos, nos EUA, demonstram que entre $60 \%$ e $80 \%$ das pessoas em tratamento psiquiátrico já foram expostas a eventos traumáticos. No México, conforme estudo realizado por Ribeiro, Andreoli, Ferri, Prince e Mari (2009), $68 \%$ da população em geral relatou ter vivenciado ao menos um evento traumático durante a vida. Diversos estudos apresentados por Kristensen, Parente e Kaszniak (2005) registram que os índices de prevalência podem variar muito, mas entre $60 \%$ e $90 \%$ da população geral é exposta a um evento potencialmente traumático ao longo da vida.

Diante de um quadro assustador que vem se perpetuando na sociedade, é preciso considerar que as vítimas desses eventos, apesar de corriqueiros, tendem a apresentar algum sofrimento psicológico (Sellbom \& Bagby, 2009; Weiner, 2007). 0 estresse gerado a partir da vivência de um evento traumático pode alterar a qualidade de vida da vítima, acarretando uma redução da motivação necessária para o desenvolvimento de atividades laborais, causando sentimentos de incompetência e, consequente, queda da autoestima (Kristensen, Schafer, \& Busnello, 2010; Miller-Burke, Attridge \& Fass, 1999).

A ideia de prejuízo psicológico parece estar vinculada à noção de trauma que, no entender de Silva (2006), é um construto decorrente da vivência de um grande estresse, podendo ocorrer como um evento isolado ou complicador de uma perturbação da personalidade existente. Tal afirmação permite pensar o trauma como desencadeado pela vivência de um evento, considerado pela vítima como terrível e que pode provocar-lhe medo. De acordo com Yehuda (2004), intenso medo ou terror são componentes essenciais em resposta a um caso de risco de vida que, além de fazer com que as pessoas reajam 
diante de uma situação de perigo, pode também gerar tensão e vir a acarretar transtornos do espectro da ansiedade.

Todavia, não obstante a carência de estudos sobre assalto no local de trabalho, por onde começar o estudo das consequências da vivência desse evento traumático? De acordo com alguns estudiosos (Luxenberg \& Levin, 2004; Miller, 2004), pesquisas que envolvam a interface da personalidade com a vivência de um trauma podem contribuir para o entendimento de fatores que determinam o curso, a expressão do comportamento e padrões de comorbidade possivelmente associados. Nesse sentido, Tarquinio, Tarquinio e Costantini (2002) afirmam que diferenças individuais na personalidade podem provocar diferentes manifestações psicológicas.

0 trauma parece ser um conceito simples, dando a ideia ampla de que, quando algo terrível acontece, a pessoa continua sentindo-se perturbada pelo evento. Entretanto, Luxenberg e Levin (2004) argumentam que o trauma é mais complexo do que essa definição, pois as respostas de cada indivíduo podem variar amplamente, dependendo da história de vida e da consequente formação da personalidade. Kaser-Boyd e Evans (2008) concordam com Luxenberg e Levin (2004) que as respostas de cada indivíduo podem assumir uma variedade de configurações, dependendo da natureza e da severidade do evento traumático e de outras variáveis como, por exemplo, o funcionamento pré-morbido da personalidade. Segundo eles, dependendo desta configuração, o trauma psicológico pode ser um fenômeno profundamente desestabilizador para aquele que o vivencia.

Na medida em que a vivência de um evento traumático como o assalto está por toda a parte, existe também a possibilidade de que os efeitos psicológicos do trauma sejam complexos e ocultos ao sujeito uma vez que se tornam corriqueiros (Kaser-Boyd \& Evans, 2008). Experiências traumáticas, convém acrescer, podem desencadear quadros de depressão, fobias, abuso de substâncias, ansiedade e pânico (Breslau et al., 1999; Foa, Keane \& Friedman, 2000; Medeiros, Kristensen \& Almeida, 2009).

Locais em que se lida com grandes quantias de dinheiro e por onde circulam muitas pessoas, como as agências bancárias e supermercados, são potenciais alvos de assaltos e, além disso, entende-se que várias pessoas podem ser atingidas ao mesmo tempo em um único evento, visto que geralmente existem diversos funcionários trabalhando quando o assalto acontece. Ademais, espera-se que, no local de trabalho, os funcionários sintam-se seguros, já que, além de ser um ambiente conhecido para essas pessoas, em geral, tais instituições são locais que contam, entre outros dispositivos de segurança, com vigilância armada, câmeras e cofre com temporizador.

Considerando que os assaltos podem atingir um grande número de pessoas e que podem ser classificados como traumáticos, por envolver uma experiência ameaçadora, questiona-se: quais as formas habituais que os funcionários assaltados no local em que exercem suas atividades laborais usam para enfrentar essa situação adversa e que aspectos da personalidade poderiam estar envolvidos nesse processo? Observa-se que, apesar de ser um tema importante e atual, parece existir uma lacuna ainda não pesquisada, isto é, trabalhos que abordam as possíveis respostas a esse tipo de trauma, considerando aspectos da personalidade e de estratégias de enfrentamento principalmente no contexto brasileiro.

Estudos que levam em conta o assalto no local de trabalho são interessantes não somente pelo aspecto social da violência urbana, mas também pelas questões psicológicas que podem estar envolvidas na vivência traumática do contexto organizacional, o qual, em princípio, oferece algum tipo de suporte e segurança. A vivência de um assalto no local de trabalho pode afetar as habilidades de enfrentamento de estresse do indivíduo devido ao sofrimento ou ao prejuízo no funcionamento social ou ocupacional e a personalidade pode estar envolvida nesse processo; sendo assim, o interesse pelo tema surge do desejo em compreender a personalidade e as reações das pessoas diante de uma situação que envolve medo e impotência.

Assim, a presente produção propõe-se a desenvolver uma revisão sistemática da literatura com o propósito de verificar o que os estudos identificados em bases de dados reconhecidas no meio acadêmico expõem a respeito da personalidade e do coping em vítimas de assalto no local em que atuam profissionalmente. A ideia que orienta essa revisão da literatura é a busca de evidências teóricas e empíricas que permitam compreender e caracterizar a sobrecarga psíquica das vítimas, considerando o evento traumático de assalto, levando em conta, mais particularmente, aspectos da personalidade e do coping. 


\section{Método}

Para atender ao objetivo estabelecido, foi operacionalizada uma revisão sistemática da literatura (Coutinho, 2003; Hulley, Cummings, Browner, Grady \& Newman, 2008) mediante a busca eletrônica de artigos indexados nas bases de dados MEDLINE/ PubMed, PsycINFO e LILACS, sendo que esta última incluiu a base SciELO e PePSIC, a partir de palavras relacionadas ao tema "personalidade" e "coping" em vítimas de assalto no local de trabalho. As consultas incluíram o período de 1995 a 2010, combinando as palavras robbery, theft, personality, coping e work usando os símbolos de truncagem “\$” (cifrão) ou “*” (asterisco) para pesquisar palavras com o mesmo radical como work, workplace ou robbery, robberies e, ainda, o operador booleano [OR] para somar à palavra robbery (theft OR robbery), ampliando o escopo da pesquisa para assalto ou roubo.

A palavra "assault", por ter fonética e grafia similar a 'assalto' no idioma português, inicialmente, foi incluída na busca dos artigos, porém esse termo foi excluído da busca final por estar relacionada a 'abuso sexual' (sendo, inclusive, cadastrada como descritor de sexual assault e sex offenses na PsycINFO) e não ao assalto como subtração de algo mediante grave ameaça ou violência à pessoa, que é o objeto principal de estudo do presente trabalho. Optou-se pelo emprego dos referidos unitermos levando-se em conta também que a maioria deles é reconhecida pelas bases de dados em questão. Além disso, esses termos têm sido usados na literatura científica especializada para abordar as questões de características de personalidade e estratégia de enfrentamento em situação de assalto no local de trabalho.

A amostra compreendeu as publicações de artigos indexados em periódicos, selecionados a partir de uma leitura prévia dos resumos. Os critérios de inclusão para seleção dos artigos foram: (1) trabalhos teóricos, empíricos ou relatos de experiência; (2) nacionais ou estrangeiros; (3) publicados entre 1995 e 2010, período de 15 anos; (4) escritos em português, inglês e espanhol; (5) amostra composta por sujeitos adultos; 6) artigos que tiveram como objeto de estudo o assalto no local de trabalho; e (7) que abordaram características de personalidade ou coping na situação de assalto em função do local de trabalho.

Adotando os critérios para inclusão das produções científicas, foi feito um levantamento preliminar através da leitura dos resumos. Com o intuito de proporcionar um tratamento mais cuidadoso dos dados, após esta etapa, foram recuperados os artigos originais na íntegra. De posse dos artigos, foi realizada então uma leitura de cada trabalho, organizando as informações de acordo com nove dimensões de análise: (1) bases de dados; (2) ano de publicação; (3) idioma da publicação; (4) país onde foi realizado o estudo; (5) tipo de produção - revisão teórica, estudo empírico ou relato de experiência; (6) método usado - quantitativo e/ou qualitativo; (7) amostra utilizada; (8) instrumentos usados para levantamento das informações; e (9) principais resultados. Dessa maneira, foi possível efetuar uma análise dos estudos selecionados a fim de se ter um panorama da produção científica acerca das características de personalidade e coping apresentadas por pessoas que foram assaltadas em seu local de trabalho.

\section{Resultados}

Foi encontrado um total de 170 artigos a partir dos descritores mencionados. A base de dados PsycINFO possibilitou identificar um total de 56 artigos; porém, excluindo os trabalhos repetidos e aqueles que não preenchiam os critérios de inclusão foram recuperados cinco. A base MEDLINE/PubMed permitiu localizar um total de 110 artigos, mas retirando os estudos repetidos e os que não preenchiam os critérios de inclusão, foi possível recuperar quatro. Já a base de dados LILACS viabilizou encontrar um total de quatro artigos, no entanto, excluindo os artigos que não preenchiam os critérios de inclusão, foi recuperado apenas um. No total, foram recuperados 10 artigos na íntegra.

A discrepância verificada entre o número de artigos inicialmente obtido (170) na busca realizada nos sistemas eletrônicos de identificação bibliográfica e a quantidade de referências considerada efetivamente pertinente (10), já era esperada em virtude do estabelecimento de critérios de inclusão bastante específicos. Cabe mencionar, contudo, que tais critérios se mostraram de extrema importância, pois segundo Hulley et al. (2008), só assim é possível selecionar adequadamente as referências inicialmente obtidas, conferindo maior precisão a esta revisão. 
Com relação ao ano e ao idioma de publicação, respectivamente segunda e terceira dimensão de análise, dos dez artigos encontrados nessa busca, a maioria dos trabalhos foi publicada no ano de 2002 (20\%) com uma maior incidência de trabalhos no idioma inglês (90\%). No que concerne à quarta dimensão de análise, o país em que foi realizado o estudo, observa-se uma maior participação de trabalhos produzidos no Canadá (30\%), seguido por duas pesquisas realizadas no Brasil (20\%). Outros países como Estados Unidos, Holanda, Dinamarca, Reino Unido e Eslovênia, para essa busca, tiveram um estudo realizado em seu país.

Quanto à quinta dimensão de análise, o tipo de produção dos artigos encontrados, constata-se que, na totalidade, são estudos empíricos. No que diz respeito à sexta dimensão, o método utilizado, apenas uma das pesquisas (Paes-Machado \& Levenstein, 2002) foi qualitativa, isto é, a maioria (90\%) dos artigos selecionados, conforme os critérios de inclusão mencionados, usaram o método quantitativo. Um resumo das informações apresentadas nas dimensões de análise um a seis, bem como a indicação do nome dos autores, é apresentado no Quadro 1.
A avaliação da sétima dimensão de análise, que diz respeito à amostra utilizada nos artigos localizados pela busca sistemática, indica que, de modo geral, os estudos selecionados, conforme os critérios de inclusão, optaram por amostras não probabilísticas por conveniência. 0 número da amostra dos dez estudos variou bastante, desde 38 sujeitos, até amostras maiores com 524 vítimas de assalto no local de trabalho. A maioria desses sujeitos foram localizados em instituições bancárias (50\%). Três estudos foram operacionalizados em diversos setores (correios, comércio, bancos) simultaneamente, mas nestes foi possível observar uma maior prevalência do número de sujeitos que atuavam no comércio, como em lojas de conveniência e supermercados. Houve um estudo efetivado somente com funcionários do comércio e outro estudo conduzido no setor de transporte coletivo.

Quanto aos instrumentos adotados pelos pesquisadores para a obtenção dos dados, observa-se que $50 \%$ dos artigos fizeram uso de um questionário elaborado especificamente para o estudo a fim de coletar dados sociodemográficos e informações relacionadas ao tema em questão. Além disso, 40\%

Quadro 1 - Resumo das informaç̃oes apresentadas nas dimensões de análise 1 a 6 e indicação do nome dos autores dos artigos e análise ( $n=10$ )

\begin{tabular}{|c|c|c|c|c|c|c|}
\hline $\begin{array}{l}\text { Base de } \\
\text { Dados }\end{array}$ & Autores & Ano Publicação & País & $\begin{array}{l}\text { Idioma } \\
\text { Artigo }\end{array}$ & $\begin{array}{c}\text { Tipo } \\
\text { Produção }\end{array}$ & Método \\
\hline LILACS & $\begin{array}{l}\text { Paes-Machado, E. \& } \\
\text { Levenstein, C. }\end{array}$ & 2002 & Brasil & Português & Empírico & Quali \\
\hline \multirow{4}{*}{$\begin{array}{l}\text { MEDLINE/ } \\
\text { PubMed }\end{array}$} & $\begin{array}{l}\text { Miller-Burke, J.; Attridge, M. \& } \\
\text { Fass, P. M. }\end{array}$ & 1999 & EUA & Inglês & Empírico & Quanti \\
\hline & $\begin{array}{l}\text { MacDonald, H. A., Colotla, V., } \\
\text { Flamer, S. \& Karlinsky, H }\end{array}$ & 2003 & Canadá & Inglês & Empírico & Quanti \\
\hline & $\begin{array}{l}\text { Seifert, A. M.; Messing, K. \& } \\
\text { Dumais, L. }\end{array}$ & 1997 & Canadá & Inglês & Empírico & Quanti \\
\hline & Richards, D. & 2000 & Reino Unido & Inglês & Empírico & Quanti \\
\hline \multirow{5}{*}{ PsycINFO } & $\begin{array}{l}\text { Kamphuis, J. H. \& Emmelkamp, } \\
\text { P. M. }\end{array}$ & 1998 & Holanda & Inglês & Empírico & Quanti \\
\hline & Elklit, A. & 2002 & Dinamarca & Inglês & Empírico & Quanti \\
\hline & $\begin{array}{l}\text { Paes-Machado, E. \& Nascimento, } \\
\text { A. M. }\end{array}$ & 2006 & Brasil & Inglês & Empírico & Quanti \\
\hline & $\begin{array}{l}\text { Bobić, J.; Pavićević, L. \& } \\
\text { Gomzi, M. }\end{array}$ & 2007 & Eslovenia & Inglês & Empírico & Quanti \\
\hline & Shercliffe, R. J. \& Colotla, V. & 2009 & Canadá & Inglês & Empírico & Quanti \\
\hline
\end{tabular}

Fonte: Dados da pesquisa. 
dos artigos empregaram escalas e inventários, sendo o mais usado o Impact of Event Scale (IES) (30\% dos artigos). Também foi feito uso de entrevistas (20\%) ou de entrevistas e observação no local de trabalho dos indivíduos participantes (20\%). Dois estudos (20\%) valeram-se da análise documental para obtenção dos dados.

No que se refere à nona dimensão de análise, os principais achados sobre personalidade e coping, foi possível verificar um maior interesse dos pesquisadores pela discussão de aspectos da personalidade. De todos os artigos selecionados a partir dos critérios de inclusão já citados, $70 \%$ abordaram características de personalidade e 30\% versaram sobre coping. Apenas dois artigos trouxeram informações tanto sobre coping quanto sobre personalidade. A respeito de personalidade, as principais características mencionadas foram relacionamento interpessoal, autoestima e afeto, as quais serão discutidas adiante.

\section{Discussão}

As bases de dados selecionadas para a procura de artigos configuraram-se como importante fonte de busca no tema investigado neste estudo, pois todas elas trouxeram trabalhos que puderam ser incluídos na revisão da literatura. Dessa forma, entende-se que os principais periódicos nacionais e internacionais no tema investigado foram acessados, apesar dos critérios de inclusão específicos definidos para esta pesquisa. 0 período de publicação foi abrangente, e assim sendo, foi possível observar que, apesar da dispersão das publicações ao longo dos anos, houve uma pequena concentração no ano de 2002 (20\%). Talvez isso se deva ao fato de que, nessa época, havia uma preocupação com o estresse ocupacional, visto que os artigos selecionados, que foram publicados entre 1998 e 2002, comentam o quanto o trabalhador assaltado tem seu labor afetado em função do estresse gerado a partir do evento.

Quanto ao idioma, foi possível observar que o inglês é o idioma mais usado (90\%) nos artigos científicos, mesmo quando o país em que foi realizado o estudo possui outro idioma como oficial. Esse fato evidencia que a escolha do idioma inglês como um dos critérios de inclusão se faz pertinente e de extrema importância. Tal resultado vai ao encontro do que Elkis (1999), assim como Frank, Coelho e Boing (2010), apontam quando constatam que o inglês vem sendo reconhecido como o idioma do mundo científico atual.

No que diz respeito ao país onde foi realizado o estudo, chama atenção o fato de o Canadá, nação a respeito da qual não se noticia número expressivo de casos de violência, ser o país que mais fez pesquisas (30\% dos artigos selecionados) sobre o tema referido. Talvez, justamente por ser um país com poucos relatos sobre violência, os assaltos no local de trabalho lá tomem outra acepção. Em nosso país, por estarmos, quem sabe, mais habituados a enfrentar esse tipo de problema, estudos sobre violência ocorrem em outras esferas, como a da violência interpessoal familiar. De qualquer forma, pesquisas que envolvam a violência no local de trabalho são importantes para que se possa oferecer a essas vítimas uma avaliação e uma intervenção adequadas, considerando o contexto em que elas estão inseridas. As questões psicológicas envolvidas em uma situação traumática vivida no contexto organizacional, em que o funcionário espera estar seguro, podem ter um conjunto de características diferente daqueles atos de violência praticados em outros locais e contextos, como é o caso da violência familiar (Kaser-Boyd \& Evans, 2008).

Corrobora essa ideia o fato de os artigos encontrados serem, na totalidade, estudos empíricos, o que evidencia o quanto os pesquisadores consideram importante explorar as variáveis selecionadas em suas pesquisas levando em conta o contexto das vítimas (Elklit, 2002; Miller-Burke et al., 1999).

Os artigos selecionados a partir das bases de dados, considerando os critérios de inclusão, evidenciaram que os pesquisadores tendem a selecionar suas amostras de pesquisa conforme conveniência do pesquisador ou das possibilidades reais de se inserir em determinados contextos laborais. A maior parte (80\%) dos artigos selecionados foi feita a partir de entrevistas e aplicação de instrumentos com mulheres e homens adultos e metade foram feitos com sujeitos assaltados em bancos (50\%), o que confirma que as instituições bancárias são visadas para a realização de assaltos. Dessa forma, conforme apontam Miller-Burke et al. (1999) e Paes-Machado e Nascimento (2006), os bancos têm reduzido a guarda de valores nas agências e, a cada dia, incentivam mais pessoas a realizarem suas operações pela internet e pelos caixas automáticos. 
Essa é uma maneira de reduzir o número de vítimas diretas de assalto.

Quanto ao tipo de local de trabalho em que ocorreu o assalto, cinco artigos utilizaram como amostra funcionários que trabalhavam em banco (Kamphuis \& Emmelkamp, 1998; Miller-Burke et al., 1999; Richards, 2000; Paes-Machado \& Nascimento, 2006; Seifert, Messing \& Dumais, 1997), três foram efetuados em setores diversos da economia, como lojas de conveniência, supermercados e correios (MacDonald, Colotla, Flamer \& Karlinsky 2003; Bobic, Pavicevic \& Gomzi, 2007; Shercliffe \& Colotla, 2009), um foi realizado somente com funcionários que atuavam no comércio (Elklit, 2002) e um entrevistou funcionários da área de transporte coletivo (Paes-Machado \& Levenstein, 2002).

Quanto aos instrumentos e técnicas usados pelos pesquisadores para obtenção das informações das amostras investigadas, observou-se que a elaboração de questionário sociodemográfico para obtenção de dados específicos é prática comum, pois foi utilizado por metade dos autores. Além disso, foi possível verificar que o uso de instrumentos de autorrelato foi a técnica mais usada para coleta de informações acerca do tema em estudo, sendo mais empregado o IES (Elklit, 2002; Kamphuis \& Emmelkamp, 1998; Richards, 2000). Esse é um instrumento de autorrelato desenvolvido por Horowitz, Wilner e Alvarez (1979) que avalia o impacto de um evento traumático a partir de sintomas do Transtorno de Estresse Pós-Traumático (TEPT).

Os instrumentos de autorrelato são de grande valia para a psicologia, visto que podem auxiliar os profissionais da área na determinação de diagnósticos e planejamento de tratamento (Reppold \& Hutz, 2008). Contudo, é importante salientar que esses instrumentos são baseados, no caso dos artigos analisados no presente estudo, no relato sobre as experiências e sintomas apresentados pelo sujeito que vivenciou o evento traumático. Tal fato pode tornar esses instrumentos de medida vulneráveis às falhas de memória, à subjetividade inerente ao sujeito avaliado e, em alguns casos, torná-los passíveis de manipulação, intencional ou inconsciente, das informações, principalmente se benefícios como indenizações e afastamento do trabalho forem desejados pelo respondente.

É interessante notar, ainda com relação aos instrumentos, que nenhum dos artigos selecionados a partir dos critérios de inclusão contempla o uso de instrumentos menos estruturados, mas que sejam padronizados. Talvez a preferência pelo uso de instrumentos de autorrelato tenha se dado por permitir aos pesquisadores o acesso a uma amostra maior, haja vista o número de sujeitos entrevistados: 524 sujeitos, entre vítimas e grupo controle no estudo de Kamphuis e Emmelkamp (1998); 123 na pesquisa de Shercliffe e Colotla (2009); 65 em Elklit (2002); e 51 em Richards (2000).

De qualquer maneira, as dificuldades vistas nos instrumentos de autorrelato podem ser minimizadas com o uso de um instrumento menos estruturado, no qual as questões específicas do trauma não sejam diretamente abordadas. Segundo Weiner (2000), tal procedimento permitiria ao avaliador acesso às experiências internas do sujeito sem que este tenha que falar diretamente sobre elas.

Quanto aos principais resultados dos estudos analisados, foi possível verificar que o foco dos artigos era a pesquisa dos sintomas apresentados após a situação de assalto. Parte desses artigos se propôs a buscar evidências sobre como um evento traumático afeta psicologicamente o trabalhador, seja com a aparição de sintomas (Bobic et al., 2007; Elklit, 2002; Kamphuis \& Emmelkamp, 1998; MacDonald et al., 2003; Miller-Burke et al., 1999; Paes-Machado \& Levenstein, 2002; Richards, 2000; Shercliffe \& Colotla, 2009) ou com evidências de alterações na personalidade (Elklit, 2002; Kamphuis \& Emmelkamp, 1998; Miller-Burke et al., 1999; Paes-Machado \& Levenstein, 2002; Paes-Machado \& Nascimento, 2006; Seifert et al., 1997; Shercliffe \& Colotla, 2009), enquanto alguns buscaram abordar também a maneira de lidar com o estresse (Elklit, 2002; Kamphuis \& Emmelkamp, 1998; MacDonald et al., 2003; Richards, 2000).

Tanto Kamphuis e Emmelkamp (1998) quanto Elklit (2002) afirmam que funcionários que experienciaram uma situação de assalto no local de trabalho apresentam maior sofrimento psicológico e reconhecem que esse sofrimento pode reduzir com o passar do tempo. De qualquer forma, o evento traumático no local de trabalho tem um impacto no indivíduo, afetando-o psicológica, emocional e fisicamente, o que vem a comprometer a eficiência no trabalho (Miller-Burke et al., 1999) e, quiçá, em outras áreas da vida. Quanto maior o número de eventos traumáticos, maior o sofrimento relatado pelas vítimas e mais suscetíveis a experimentarem sintomas (Elklit, 2002; Kamphuis \& Emmelkamp, 
1998). Além disso, a culpa que algumas vítimas sentem pelo acontecimento do assalto sugere, nos estudos de Paes-Machado e Nascimento (2006) e de Shercliffe e Colotla (2009), uma queda na autoestima e a consequente presença de sintomas.

0 estudo de Elklit (2002), realizado poucos dias após o assalto, assim como o de Kamphuis e Emmelkamp (1998), que foi conduzido entre seis meses e um ano após o evento, destacam que o assalto é um interjogo dinâmico em que diferentes participantes atuam e uma série de ações acontece. Elklit (2002) indica que geralmente uma pessoa que experiencia uma situação de assalto também vivenciou outros eventos traumáticos ao longo da vida e, portanto, cada indivíduo terá suas próprias reações psicológicas. Nesse sentido, Paes-Machado e Levenstein (2002) sugerem uma intervenção logo após o assalto a fim de recuperar a autoestima das vítimas e, assim, terem a oportunidade de um maior controle do estresse.

Considerando as características individuais, ou seja, cada um terá suas próprias reações, Kamphuis e Emmelkamp (1998) sugerem que as respostas após o assalto e a personalidade pré-existente têm relação com o processamento emocional do assalto. Acrescentam ainda que a vivência de outros eventos traumáticos ao longo da vida vai formando a personalidade do sujeito e, a partir disso, a leitura subjetiva que o sujeito faz do assalto é única, apresentando diferentes graus de dificuldade para selecionar a estratégia de enfrentamento mais adequada. Com a intenção de explicar tal definição, esses autores exemplificam que algumas vítimas de assalto pesquisadas em seu estudo apresentaram altos níveis de sofrimento psicológico, contudo, um grupo não demonstrou níveis tão altos. Segundo eles, este achado revela a importância das características individuais no processamento emocional do assalto.

Ainda pensando sobre as formas de lidar com o estresse, Elklit (2002) aborda o tema do coping avaliando as defesas psicológicas em indivíduos assaltados. Ele entende que um evento traumático pode enfraquecer as defesas psíquicas de uma pessoa e indica que, em seu estudo, foi encontrado um maior número de defesas imaturas que o apontado em outro estudo sobre violência. Porém, refere o autor, essa informação pode ter sido distorcida pelo fato de ter usado para comparação uma pesquisa com pais que perderam seus filhos e que a idade destes sujeitos era maior que a idade da amostra de 65 funcionários do comércio dinamarquês pesquisada em seu estudo. Sem analisar o impacto pertinente a cada evento, o assalto parece mais corriqueiro do que a perda de um filho. Além disso, as vítimas do estudo realizado por Elklit (2002) receberam bom suporte familiar e de amigos. De acordo com os dados colhidos, o contato com outras vítimas em situações similares e as atitudes em relação ao futuro, à própria capacidade e a outras pessoas foram consideradas pelo autor como bastante positivas.

Nesse sentido, alguns dos 141 funcionários do estudo de Miller-Burke et al. (1999) referiram que seus relacionamentos, tanto no ambiente de trabalho quanto na vida pessoal, pioraram. Todavia, algumas pessoas informaram que seus relacionamentos melhoraram. Os autores do artigo entendem que o fato de ter recebido apoio de familiares e amigos pode ter contribuído para a melhora no relacionamento de alguns. Interessante que, nesse estudo realizado nos Estados Unidos, o relacionamento interpessoal não foi correlacionado com outras dimensões analisadas como os sintomas, por exemplo.

Analisando as respostas após assalto, Kamphuis e Emmelkamp (1998) e MacDonald et al. (2003) ponderam que o sofrimento psicológico pode ser associado com a experiência subjetiva do evento. No estudo realizado por Kamphuis e Emmelkamp (1998), envolvendo 310 vítimas de assalto no local de trabalho, o sofrimento verificado pode ser associado com a estratégia de coping evitativa subsequente ao assalto, com a vivência de outros eventos traumáticos e com a autoestima presente antes do assalto. Segundo esses autores, os resultados sugerem que a autoestima pode fornecer uma espécie de "amortecedor" contra o impacto do evento.

0 sofrimento presente na amostra do estudo de Elklit (2002) apareceu no uso das estratégias de coping, sendo o estilo evitativo também o mais comum. Esse autor assinala que o estilo de enfrentamento mais usado foi o evitativo, porém, quando ele não era suficiente, as pessoas tendiam a fazer algo para evitar o pensamento, o sentimento ou reviver o evento. Além disso, tanto o coping emocional quanto o coping evitativo foram positivamente associados com o coping racional, o que foi analisado pelo autor como uma fase caótica de adaptação em que o sujeito lança mão de diferentes estratégias ao mesmo tempo para dar conta da demanda. Assim como 
no trabalho de Kamphuis e Emmelkamp (1998), o artigo apresentado por Elklit (2002) informa que o uso da estratégia de coping evitativa foi associado com sintomas de estresse agudo. $\mathrm{O}$ autor assinala que uma intervenção, logo após o assalto, que encoraje o uso de estratégias focadas na emoção e no problema pode ajudar a prevenir sofrimento.

Além da personalidade e do coping, os trabalhos analisados reconheceram que: a idade não influenciou o nível de trauma percebido e o tipo de sintomas apresentados após o assalto (Miller-Burke et al., 1999); as mulheres apresentaram mais sintomas de somatização que os homens (Elklit, 2002; Kamphuis \& Emmelkamp, 1998); a saúde física piorou (Miller-Burke et al., 1999) e sintomas de estresse pós-traumático estavam presentes (Elklit, 2002; Kamphuis \& Emmelkamp, 1998; Miller-Burke et al., 1999). Contudo, quanto maior o nível educacional, menor a ocorrência de sintomas psicológicos (Elklit, 2002).

Similar ao trabalho de Kamphuis e Emmelkamp (1998), a pesquisa de Elklit (2002) apontou que vítimas que vivenciaram outros eventos traumáticos ao longo da vida apresentaram mais sintomas. No referido estudo, os sintomas apresentados foram associados ao nível de percepção de ameaça durante o assalto, um estilo de enfrentamento depressivo/evitativo, assim como com o impacto de outras vivências traumáticas. Já Miller-Burke et al. (1999) identificaram que a severidade dos sintomas não está relacionada com a idade, o gênero, o cargo ou o tempo entre assalto e avaliação, acrescentando que a severidade dos sintomas em parte depende da leitura que o sujeito faz do evento. Esses autores entendem que a intervenção após o incidente deve incluir estratégias de enfrentamento que possam reduzir o impacto do evento traumático.

\section{Considerações finais}

A presente revisão da literatura permitiu traçar um panorama dos estudos que investigaram personalidade e estratégias de enfrentamento em funcionários assaltados no local de trabalho. Apesar da escassa literatura disponível, foi possível verificar potencialidades de estudos empíricos, a partir de evidências procedentes de referências confiáveis. Tal constatação evidencia que, apesar de corriqueiro, o assalto também tem efeitos no psiquismo das vítimas e, por isso, não pode ser deixado de lado. $\mathrm{O}$ assalto no local de trabalho tem um impacto na saúde, na vida pessoal e profissional da vítima.

De acordo com a revisão realizada, as pessoas tendem a usar diferentes formas de enfrentamento do estresse gerado a partir da vivência traumática. 0 que parece predizer o sofrimento psicológico após o assalto é a vivência de outros eventos traumáticos visto que pode influenciar a formação da personalidade através da autoestima, da dificuldade no relacionamento interpessoal, da maneira como processa o afeto e do uso de estratégias de $\mathrm{Co}^{-}$ ping diferentes e de modo alternado numa mesma situação. Além disso, quanto à personalidade, nesta revisão foi possível identificar a presença de evidências de algumas dificuldades no relacionamento interpessoal, no processamento do afeto e redução da autoestima nas vítimas. No que diz respeito à estratégia de enfrentamento, observou-se que a estratégia mais usada pelas vítimas foi a evitativa, porém o indício de maior sofrimento estava nas vítimas que usavam estratégias diferentes de modo alternado.

Trabalhadores de instituições que lidam com grandes quantias de dinheiro e trabalham com acesso fácil e direto do público são expostos a um contexto de potencial risco de assalto, o que os torna vulneráveis ao sofrimento psíquico devido à violência interpessoal comunitária. Parece oportuno, portanto, empregar esforços para averiguar aspectos relacionados ao estilo de enfrentamento do estresse e personalidade, principalmente características como afeto, autoestima e relacionamento interpessoal nas vítimas de assalto no local de trabalho para que se possa planejar uma intervenção adequada.

0 número de artigos encontrados que constituíram o corpo de análise desta revisão serve como ilustração da produção científica sobre o tema. Levando-se em conta o descarte de artigos que se repetiram ou não preencheram os critérios de inclusão estabelecidos para a busca realizada, pode-se afirmar que os artigos analisados constituem, portanto, um recorte que ajuda a entender o tema pesquisado. Esse fato não descarta a possibilidade de haver maior incidência de estudos referentes ao tema assalto no local de trabalho durante os anos abrangidos pela presente revisão, porém não estruturados de acordo com o enfoque deste estudo. 


\section{Referências}

American Psychiatric Association, [APA], (2002). Manual diagnóstico e estatístico de transtornos mentais DSM-IV-TR. Porto Alegre: Artmed.

Bobic, J., Pavicevic, L., \& Gomzi, M. (2007). Post-traumatic stress disorder in armed robbery victims and ex-prisoners of war. Studia Psychologica, 49(2), 135-144.

Brasil (2000). Ministério da Justiça. Código Penal Brasileiro. São Paulo: Editora Revista dos Tribunais.

Breslau, N., Chilcoat, H., Kessler, R., \& Davis, G. (1999). Previous exposure to trauma and PTSD effects of subsequent trauma: Results from the Detroit Area Survey of Trauma. American Journal, 156, 902-907.

Coutinho, E. S. (2003). Meta-Análise. In R. A. Medronho, D. M. Carvalho, K. V. Bloch, R. R. Luiz \& G. L. Werneck, Epidemiologia, (pp. 447-455). São Paulo: Atheneu.

Elkis, H. (1999). Fatores de impacto de publicações psiquiátricas e produtividade científica. Revista Brasileira de Psiquiatria, 21(4), 231-236. doi:10.1590/ S1516-44461999000400012.

Elklit, A. (2002). Acute stress disorder in victi$\mathrm{ms}$ of robbery and victims of assault. Journal of Interpersonal Violence, 17(8), 872-887. doi:10.1177/0886260502017008005

Foa, E., Keane, T., \& Friedman, M. (2000). Guidelines for treatment of PTSD. Journal of Traumatic Stress, 13(4), 539-555. doi:10.1023/A:1007802031411.

Frank, S., Coelho, E., \& Boing, A. (2010). Perfil dos estudos sobre violência contra a mulher por parceiro íntimo: 2003 a 2007. Revista Panamericana de Salud Pública, 27(5), 376-381. doi:10.1590/ S1020-49892010000500008.

Horowitz, M., Wilner, N., \& Alvarez, W. (1979). Impact of Events Scale: A measure of subjective stress. Psychosomatic Medicine, 41, 209-218. PMid:472086.

Hulley, S. B., Cummings, S. R., Browner, W. S., Grady, D. G., \& Newman, T. B. (2008). Delineando a pesquisa clínica - Uma abordagem epidemiológica. Porto Alegre: Artmed.
Kamphuis, J. H., \& Emmelkamp, P. M. (1998). Crime-related trauma: Psychological distress in victims of bankrobbery. Journal of Anxiety Disorders, 12(3), 199-208. doi:10.1016/S0887-6185(98)00009-7.

Kaser-Boyd, N., \& Evans, F. (2008). Rorschach assessment of psychological trauma. In C. Gacono \& F. Evans. The Handbook of Forensic Rorschach Assessment. (pp. 255-277). New York: Routledge.

Kristensen, C., Parente, M., \& Kaszniak, A. (2005). Transtorno de estresse pós-traumático: Critérios diagnósticos, prevalência e avaliação. In R. Caminha. Transtorno do Estresse Pós-Traumático (TEPT): Da neurobiologia à terapia cognitiva. (pp. 15-35). São Paulo: Casa do Psicólogo.

Kristensen, C., Schafer, L., \& Busnello, F. (2010). Estratégias de coping e sintomas de stress na adolescência. Estudos de Psicolgia, 27(1), 21-30. doi:10.1590/ S0103-166X2010000100003.

Krug, E. G., Dahlberg, L. L., Mercy, J. A., Zwi, A. B., \& Lozano, R. (2002). Relatório Mundial sobre a Violência e a Saúde. Genebra: World Health Organization.

Luxenberg, T., \& Levin, P. (2004). The role of the Rorschach in the assessment and treatment of trauma. In J. Wilson, \& T. Keane Assessing psychological trauma and PTSD. (pp. 190-225). New York: Guilford.

MacDonald, H., Colotla, V., Flamer, S., \& Karlinsky, H. (2003). Posttraumatic Stress Disorder (PTSD) in the workplace: A descriptive study of workers experiencing PTSD resulting from work injury. Journal of Occupational Rehabilitation, 13(2), 63-77. doi:10.1023/A:1022563930482.

Medeiros, L., Kristensen, C., \& Almeida, R. (2009). Estresse pós-traumático em pacientes vítimas de queimaduras: uma revisão da literatura. Aletheia, 29, 177-189.

Miller, M. (2004). Personality and the development and expression of PTSD. PTSD Research Quarterly, 15(3), 1-8.

Miller-Burke, J., Attridge, M., \& Fass, P. (1999). Impact of traumatic events and organizational response: A study of bank robberies. Journal of Occupational and Environmental Medicine, 41(2), 73-83. doi:10.1097/00043764-199902000-00001. 
Paes-Machado, E., \& Levenstein, C. (2002). Assaltantes a bordo: Violência, insegurança e saúde no trabalho em transporte coletivo de Salvador, Bahia, Brasil. Cadernos de Saúde Pública, 18(5), 1215-1227. doi:10.1590/S0102-311X2002000500014.

Paes-Machado, E., \& Nascimento, A. M. (2006). Bank money shields: Work-related victimisation, moral dilemmas and crisis in the bank profession. International Review of Victimology, 13(1), 1-25. doi:10.1177/026 975800601300101.

Reppold, C., \& Hutz, C. (2008). Investigação psicodiagnóstica de adolescentes: Encaminhamentos, queixas e instrumentos utilizados em clínicas-escolas. Avaliação Psicológica, 7(1), 85-91.

Ribeiro, W. S., Andreoli, S. B., Ferri, C. P., Prince, M., \& Mari, J. J. (2009). Exposição à violência e problemas de saúde mental em países em desenvolvimento: Uma revisão da literatura. Revista Brasileira de Psiquiatria, 31(2), 49-57.

Richards, D. (2000). Symptom severity, personal and social variables after armed robbery. British Journal of Clinical Psychology, 39(4), 415-419. doi:10.1348/014466500163419.

Seifert, A., Messing, K., \& Dumais, L. (1997). Star wars and strategic defense initiatives: Work activity and health symptoms of unionized bank tellers during work reorganization. International Journal of Health Services, 27(3), 455-477. doi:10.2190/7KUK-2D4L-0G1N-8DNY.
Sellbom, M., \& Bagby, M. R. (2009). Identifying PTSD personality subtypes in a workplace trauma sample. Journal of Traumatic Stress, 22(5), 471-475. doi:10.1002/jts.20452.

Shercliffe, R. J., \& Colotla, V. (2009). MMPI-2 profiles in civilian PTSD: An examination of differential responses between victims of crime and industrial accidents. Journal of Interpersonal Violence, 24(2), 349-360. doi:10.1177/0886260508316482.

Silva, F. C. (2006). Ameaças à Infância: Do trauma psíquico ao transtorno de estresse pós-traumático. Dissertação, Mestrado em Psicologia, Pontifícia Universidade Católica de São Paulo, São Paulo-SP.

Tarquinio, C., Tarquinio, P., \& Costantini, M. L. (2002). Identity alteration and psychotraumatic impact of violence situations in a professional context. European Review os Apllied Psychology, 52(2), $135-148$.

Weiner, I. B. (2000). Princípios da Interpretação do Rorschach. São Paulo: Casa do Psicólogo.

Weiner, I. B. (2007). Rorschach assessment in forensic cases. In A. Goldstein, Forensic Psychology: Emerging topics and expanding roles, (pp. 127-153). New Jersey: John Wiley \& Sons.

Yehuda, R. (2004). Risk and resilience in posttraumatic stress disorder. Journal of Clinical Psychiatry, 65(1), 29-36. PMid:14728094. 